\title{
Immunogenicity and safety of a live herpes zoster vaccine in hematopoietic stem cell transplant recipients
}

June Young Chun ${ }^{1,2 \dagger}$, Kichun Kim ${ }^{1 \dagger}$, Min Kyeong Lee ${ }^{1}$, Chang Kyung Kang ${ }^{1}$, Youngil Koh', Dong-Yeop Shin ${ }^{1}$, Junshik Hong ${ }^{1}$, Pyoeng Gyun Choe ${ }^{1}$, Nam Joong Kim¹, Sung-Soo Yoon ${ }^{1}$, Wan Beom Park ${ }^{1 *}$, Inho Kim ${ }^{1 *}$ and Myoung-don $\mathrm{Oh}^{1}$

\begin{abstract}
Background: Herpes zoster (HZ) infection of hematopoietic stem cell transplant (HSCT) patients is of clinical concern. Vaccination could help restore immunity to varicella zoster virus (VZV); however, temporal changes in immunogenicity and safety of live HZ vaccines after HSCT is still unclear. The aim of this study was to elucidate the temporal immunogenicity and safety of the HZ vaccine according to time since HSCT and to determine optimal timing of vaccination.

Methods: Live HZ vaccine was administered to patients 2-5 years or $>5$ years post-HSCT. Control groups comprised patients with a hematologic malignancy who received cytotoxic chemotherapy and healthy volunteers. Humoral and cellular immunogenicity were measured using a glycoprotein enzyme-linked immunosorbent assay (gpELISA) and an interferon- $\gamma$ (IFN- $\gamma$ ) enzyme-linked immunospot (ELISPOT) assay. Vaccine-related adverse events were also monitored.

Results: Fifty-six patients with hematologic malignancy (41 in the HSCT group and 15 in the chemotherapy group) along with 30 healthy volunteers were enrolled. The geometric mean fold rises (GMFRs) in humoral immune responses of the 2-5 year and $>5$ year HSCT groups, and the healthy volunteer group, were comparable and significantly higher than that of the chemotherapy group $(3.15,95 \% \mathrm{Cl}$ [1.96-5.07] vs 5.05, 95\% Cl [2.50-10.20] vs 2.97, 95\% Cl [2.30-3.83] vs 1.42,95\% Cl [1.08-1.86]). The GMFR of cellular immune responses was highest in the HSCT 2-5 year group and lowest in the chemotherapy group. No subject suffered clinically significant adverse events or reactivation of VZV within the follow-up period.
\end{abstract}

Conclusion: Our findings demonstrate that a live $\mathrm{HZ}$ vaccine is immunogenic and safe when administered 2 years post-HSCT.

Keywords: Herpes zoster, Varicella zoster virus, Hematopoietic stem cell transplantation, Immunogenicity, Safety

\footnotetext{
* Correspondence: wbpark1@snu.ac.kr; ihkimmd@snu.ac.kr

†June Young Chun and Kichun Kim contributed equally to this work. ${ }^{1}$ Department of Internal Medicine, Seoul National University College of Medicine, 101 Daehak-ro, Jongno-gu, Seoul 03080, Republic of Korea Full list of author information is available at the end of the article
} 


\section{Background}

Herpes zoster (HZ), also called shingles (derived from Latin cingulus meaning girdle), is a dermatomalvesicular disease associated with severe pain [1]. It is caused by reactivation of latent varicella zoster virus (VZV) within sensory ganglia and is more common in immunocompromised patients [1]. The incidence of $\mathrm{HZ}$ increases with age; the highest incidence (5-10 cases per 1000 persons) occurs in the sixth decade or beyond [2]. The burden of $\mathrm{HZ}$ for those with a hematopoietic stem cell transplant (HSCT) is $20-53 \%$ overall; the greatest risk (94 cases per 1000 person-years) occurs within 2 years of HSCT [3-5].

There are no clear guidelines regarding live vaccination after HSCT. With respect to HZ vaccines, limited data support vaccination after HSCT due to concerns about vaccine-induced VZV infection and lack of evidence regarding vaccine-induced immunogenicity [6-8]. Vaccination against $\mathrm{HZ}$ might be considered only when 24 months have elapsed since HSCT, and only in recipients showing no signs of graft-versus-host disease (GvHD) or relapse, and in those not taking immunosuppressants [9-11]. The most recent guidelines from the 2017 European Conference on Infections in Leukaemia (ECIL 7) oppose administration of live $\mathrm{HZ}$ vaccines; instead, they recommend antiviral agents to prevent VZV reactivation [12]. However, even with prolonged administration of antiviral agents, the incidence of $\mathrm{HZ}$ increases after discontinuation of prophylaxis $[4,13]$.

Although necessary, there is not enough evidence to support a minimum interval between transplantation and vaccination. A theoretical minimum of 24 months supposes that the HSCT recipient is immunocompetent 2 years after HSCT [14]. Here, we assessed (i) the temporal immunogenicity of live $\mathrm{HZ}$ vaccine in post-HSCT and (ii) the safety of live $\mathrm{HZ}$ vaccine in these patients.

\section{Methods}

\section{Study design}

This was a clinical study conducted at Seoul National University Hospital (SNUH), which is a tertiary care university-affiliated hospital in South Korea.

From July 2017 to August 2018, we prospectively enrolled patients with a hematologic malignancy who had survived with either autologous or allogeneic HSCT. Additional inclusion criteria were as follows: age older than 50 years and provision of informed consent for participation. Exclusion criteria included GvHD, use of immunosuppressants or antiviral agents, HZ reactivation within 1 year of the study period, or receipt of $\mathrm{HZ}$ vaccines. These patients were stratified according to time since transplantation: $2-5$ years and $>5$ years (hereafter referred to as HSCT $2-5 \mathrm{yr}$ and $\mathrm{HSCT}>5 \mathrm{yr}$, respectively).
Controls included patients with a hematologic malignancy who had undergone cytotoxic chemotherapy and survived without relapse for at least 6 months before enrollment (referred to as the chemotherapy group). Inclusion and exclusion criteria were applied in the same manner as for the HSCT groups. Lastly, healthy volunteers aged > 50 years without recent $\mathrm{HZ}$ reactivation within 1 year were recruited (referred to as the healthy group).

Study participants were given a single dose $(0.65 \mathrm{~mL})$ of ZOSTAVAX ${ }^{\bullet}$. Blood samples were collected to test both humoral and cellular immune responses against VZV prior to vaccination and at 6 weeks postvaccination. Baseline characteristics included age, sex, underlying diseases, type of HSCT or cytotoxic chemotherapy, and previous history of $\mathrm{HZ}$.

\section{Glycoprotein ELISA (gpELISA)}

Although correlates of protection (CoP) for $\mathrm{HZ}$ vaccines have not been defined clearly, fold rises in antibody titers in the glycoprotein enzyme-linked immunosorbent assay (gpELISA) are thought to be an excellent immune correlate of protection [15]. VZV-specific antibodies were measured quantitatively using a SERION ELISA classic Varicella Zoster Virus IgG kit (Institut Virion/ Serion GmbH, Würzburg, Germany). This gpELISA assay uses a lentil-lectin affinity-purified preparation of glycoprotein from VZV-infected MRC-5 cells as the antigen [16, 17]. Antigen-coated 96-well plates were incubated with test sera. Human immunoglobulin G antibodies (IgGs) bound to antigen were detected by incubation with anti-human IgG antibodies. Color was developed after reaction with a substrate. Optical density was read at $405 \mathrm{~nm}$.

\section{ELISPOT assay}

Peripheral blood mononuclear cells (PBMCs) collected and frozen during the study period were tested using a $\mathrm{BD}^{\mathrm{m}}$ IFN- $\gamma$ ELISPOT kit (BD Bioscience, San Jose, CA, USA). Briefly, 96-well plates were coated with $100 \mu \mathrm{L}$ of an anti-human-IFN- $\gamma$ antibody at a concentration of $5 \mu \mathrm{g} / \mathrm{mL}$ overnight at $4{ }^{\circ} \mathrm{C}$. Next, $1 \times 10^{6} \mathrm{PBMCs} / 100 \mu \mathrm{L} /$ well were activated with $5 \mu \mathrm{g} / \mathrm{mL}$ phytohemagglutinin (mitogen), UV-inactivated VZV culture supernatants (at a dilution of $1: 80$ ) and mock antigen supernatants for $16-20 \mathrm{~h}$ at $37^{\circ} \mathrm{C}$ in a $5 \% \mathrm{CO}_{2}$ humidified incubator [18]. After washing, a solution containing a biotinylated antihuman-IFN- $\gamma$ detection antibody was added to each well, and streptavidin-HRP solution and substrate were used for color development. Spots were counted with a CTL-ImmunoSpot ${ }^{\circ}$ reader (CTL ImmunoSpot, Cleveland, $\mathrm{OH}, \mathrm{USA}$ ) and reported as the net number of VZV-specific IFN- $\gamma$ spot-forming cells (sfc) per $10^{6}$ PBMCs (the difference between responses to VZV 
antigen and control antigen) [19]. Samples lacking sufficient PMBCs and results with phytohemagglutinin responses $<300 \mathrm{sfc}$ were not included in the analysis [20].

\section{Safety}

Vaccine adverse events (AEs) were monitored based on spontaneous reports from participants, subject daily review, and history taking by investigators. All vaccinated individuals were requested to inform the study nurse or physician immediately if they noticed any serious AEs after vaccination. The participants were also requested to fill in a self-reported structured questionnaire up to 6 weeks post-vaccination and submit it on the second visit. The type and severity of local and systemic adverse events were assessed again by the study nurse 6 weeks after vaccination. Adverse events were graded on a standard scale [21]. The causality of an adverse event after immunization was classified as follows: unlikely (inconsistent), possible (indeterminate), or likely (consistent) [22].

\section{Statistical analysis}

For continuous variables, mean (standard deviation, SD) and median (interquartile range, IQR) were used for normally and abnormally distributed data. Categorical variables were expressed as numbers and percentages. A $t$-test was used to compare continuous variables and the Chi-square or Fisher's exact test was used to compare categorical variables. One-way ANOVA with Dunnett's adjustment or Kruskal-Wallis test was used to calculate $P$-values for comparisons of more than two variables. All tests were two-sided and $P$-values $<0.05$ were considered statistically significant. All statistical analyses were performed using SPSS for Windows (version 22; IBM Corp., Armonk, NY, USA).

\section{Results}

\section{Baseline characteristics}

The study screened 60 hematology patients at baseline; four patients dropped out due to follow-up loss $(n=3)$ and disease recurrence $(n=1)$. Among the remaining 56 subjects, 26 were assigned to the HSCT $2-5 \mathrm{yr}$ group and 15 to the HSCT $>5 \mathrm{yr}$ group. The chemotherapy group included 15 patients. In addition, 30 healthy volunteers were enrolled (Fig. 1).

Participants in each group were balanced by age; however, the male-to-female ratio of the healthy group was lower (1:2) than that in the other groups (Table 1). Leukemia and lymphoma were the most common ( $>50 \%$ of participants in each group) underlying malignancies. Twenty patients in the HSCT 2-5 yr group (77\%), and nine in the HSCT > $5 \mathrm{yr}$ group $(60 \%)$ underwent allogeneic HSCT. A history of $\mathrm{HZ}$ reactivation was documented in study groups. Twelve patients in the HSCT $2-5 \mathrm{yr}$ group (46\%), nine in the HSCT $>5 \mathrm{yr}$ group $(60 \%)$, and six in the chemotherapy group $(40 \%)$ had shingles before enrollment in the study (Table 1). Among those reactivated, nine in the HSCT $2-5 \mathrm{yr}$ group (75\%), six in the HSCT $>5$ yr group (67\%), and five in the chemotherapy group (83\%) had history within 2 years after HSCT or last cytotoxic chemotherapy.

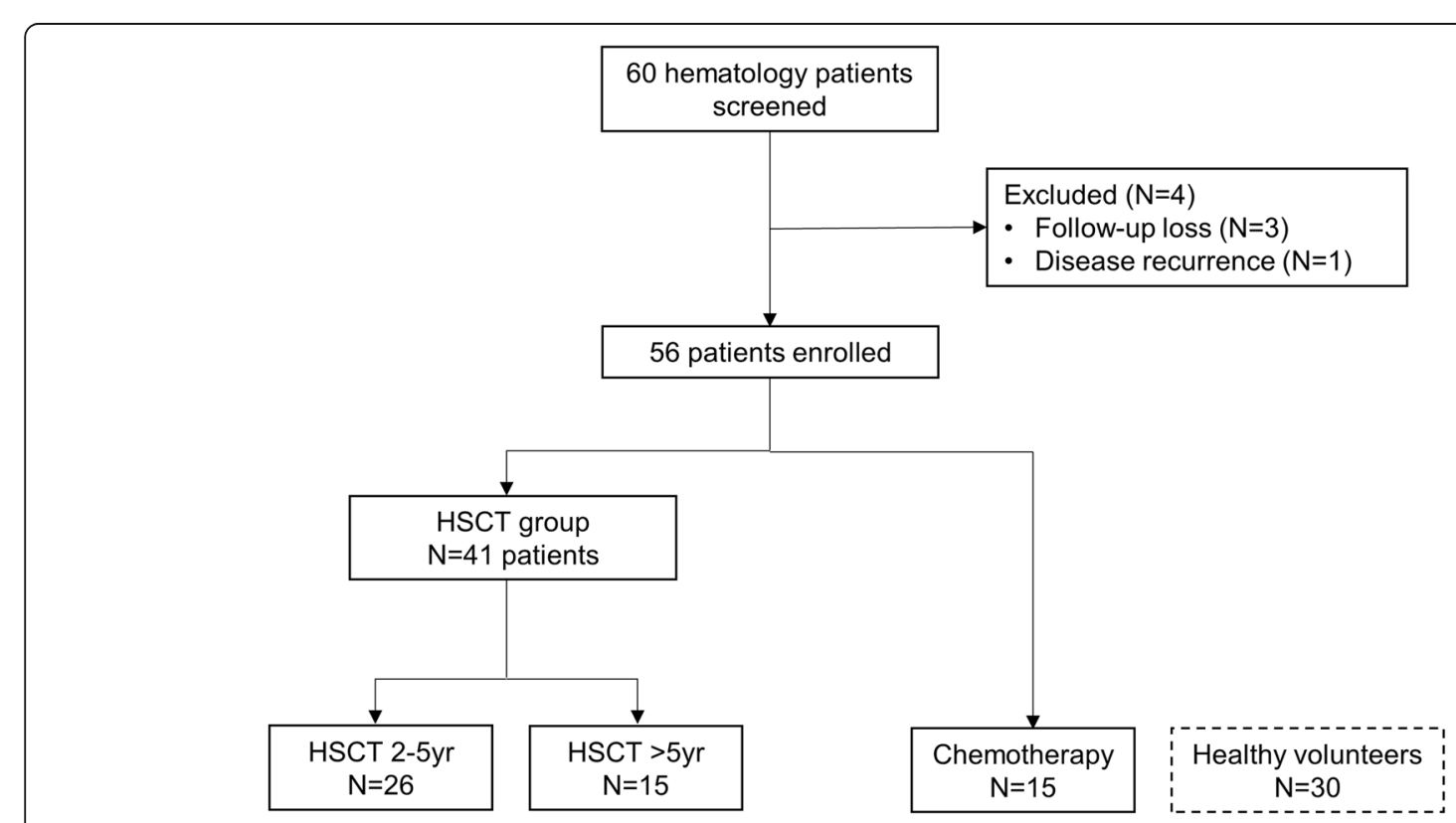

Fig. 1 Flow diagram of study enrollment. HSCT, hematopoietic stem cell transplantation; N, number 
Table 1 Baseline Characteristics

\begin{tabular}{|c|c|c|c|c|}
\hline & $\begin{array}{l}\text { HSCT } 2-5 \mathrm{yr} \\
(n=26)\end{array}$ & $\begin{array}{l}\text { HSCT }>5 \mathrm{yr} \\
(n=15)\end{array}$ & $\begin{array}{l}\text { Chemotherapy } \\
(n=15)^{c}\end{array}$ & $\begin{array}{l}\text { Healthy } \\
(n=30)\end{array}$ \\
\hline Median age, yr (IQR) & $60(55-63)$ & $58(53-62)$ & $64(57-69)$ & $61(57-66)$ \\
\hline Male sex, n (\%) & $15(58)$ & $9(60)$ & $10(67)$ & $10(33)$ \\
\hline \multicolumn{5}{|l|}{ HSCT indication, n (\%) } \\
\hline Leukemia & $11(42)$ & $4(27)$ & $14(93)$ & N/A \\
\hline Lymphoma & $2(8)$ & $7(47)$ & & \\
\hline Multiple myeloma & $6(23)$ & $1(6.5)$ & & \\
\hline Myelodysplastic syndrome & $4(15)$ & $2(13)$ & & \\
\hline Others $^{a}$ & $3(12)$ & $1(6.5)$ & $1(7)$ & \\
\hline \multicolumn{5}{|l|}{ Type of HSCT, n (\%) } \\
\hline Allogeneic & $20(77)$ & $9(60)$ & N/A & N/A \\
\hline Autologous & $6(23)$ & $6(40)$ & & \\
\hline Time between HSCT or last chemo and the study date, mo (IQR) & $35(25-44)$ & $87(66-91)$ & $65(23-104)$ & N/A \\
\hline Previous shingles history, yes, $\mathrm{n}(\%)$ & $12(46)$ & $9(60)$ & $6(40)$ & $0(0)$ \\
\hline Shingles within 2 yrs after HSCT or last chemo, $\mathrm{n}(\%)$ & $9 / 12(75)$ & $6 / 9(67)$ & $5 / 6(83)$ & N/A \\
\hline Median years from shingles to the study date (IQR) & $3(1.5-4)^{b}$ & $5(5-7)$ & $2.5(1.3-6)$ & N/A \\
\hline
\end{tabular}

IQR Interquartile range, yr Year, mo Month, N/A Not applicable, chemo Chemotherapy

${ }^{a}$ Aplastic anemia $(n=3)$, Myeloid sarcoma $(n=1)$, Granulocytic sarcoma $(n=1)$

b1 patient could not remember the exact time of shingles

${ }^{\mathrm{C}}$ All but one patient had acute myeloid leukemia. None had received targeted monoclonal antibody therapies

\section{Humoral immune responses}

At baseline, the gpELISA geometric mean titers (GMTs) in the HSCT 2-5 yr, chemotherapy, and healthy groups were similar (841.08, 95\% CI [439.58-1609.29] vs 515.92 , 95\% CI [302.03-881.28] vs 657.15 , 95\% CI [424.18-1018.09]); values were significantly lower in the HSCT > 5 yr group (262.89, 95\% CI [149.59-462.00], $p=0.041)$. At 6 weeks post-vaccination, immune responses measured in the gpELISA increased significantly in all four groups, compared with the baseline (Fig. 2a, Supplementary Table 1). The GMFR in the chemotherapy group was significantly lower than in the other groups (1.42; 95\% CI, 1.08-1.86) (Fig. 2c). The GMFRs of the HSCT $2-5 \mathrm{yr}, \mathrm{HSCT}>5 \mathrm{yr}$, and healthy groups were not significantly different $(3.15,95 \%$ CI [1.96-5.07] vs 5.05 , 95\% CI $[2.50-10.20]$ vs $2.97,95 \%$ CI [2.303.83]) (Fig. 2c). Patients with a history of shingles showed higher baseline gpELISA GMTs than those without, but the GMFRs were uninfluenced (Supplementary Table 2).

\section{Cellular immune responses}

The ELISPOT assay was performed in only 52 subjects due to lack of qualified blood samples (HSCT 2-5 yr $[n=12]$, HSCT $>5$ yr $[n=7]$, chemotherapy $[n=13]$, and healthy group $[n=20])$. At baseline, the geometric mean concentrations (GMCs) of IFN- $\gamma$-secreting VZVspecific PBMCs were higher in the healthy group (51.39, 95\% CI [34.36-76.88], $p=0.006)$ than in the other three groups $(9.00,95 \% \mathrm{CI}[3.43-23.60]$ vs $27.99,95 \% \mathrm{CI}$ [8.45-92.65] vs 14.76, 95\% CI [6.57-33.16]). Six weeks post-vaccination, the GMCs of IFN- $\gamma$-secreting VZV-specific PBMCs increased significantly in all groups (Fig. 2b, Supplementary Table 1). The GMFR of the IFN- $\gamma$ ELISPOT assay was highest in the HSCT $2-5$ yr group $(8.39,95 \%$ CI [3.3021.32]) and lowest in the chemotherapy group (1.64, 95\% CI [1.23-2.17]) (Fig. 2d). The GMFRs in the HSCT > $>\mathrm{yr}$ and healthy groups were 5.08 (95\% CI, [1.86-13.86]) and 1.82 (95\% CI, [1.32-2.49]), respectively. Patients with a history of shingles tended to have higher baseline GMCs than those without, but the GMFRs were uninfluenced (Supplementary Table 3).

\section{Safety}

There were no reported cases of VZV reactivation up to 6 weeks post-vaccination in any of the study groups. Injection site reactions such as local pain, redness, edema, and itching were the most common adverse events reported by vaccine recipients (Table 2). Three patients in the HSCT $2-5$ yr group $(11.5 \%)$ and one each in the HSCT $>5$ yr $(6.6 \%)$ and chemotherapy (6.6\%) groups reported vaccine-related systemic adverse events such as headache, myalgia, and fatigue (Table 2). All adverse events were mild (grade 1) and occurred within 7 days of vaccination.

\section{Discussion}

$\mathrm{HZ}$ is a high burden of disease among HSCT recipients whose immune status has been shut down and then 

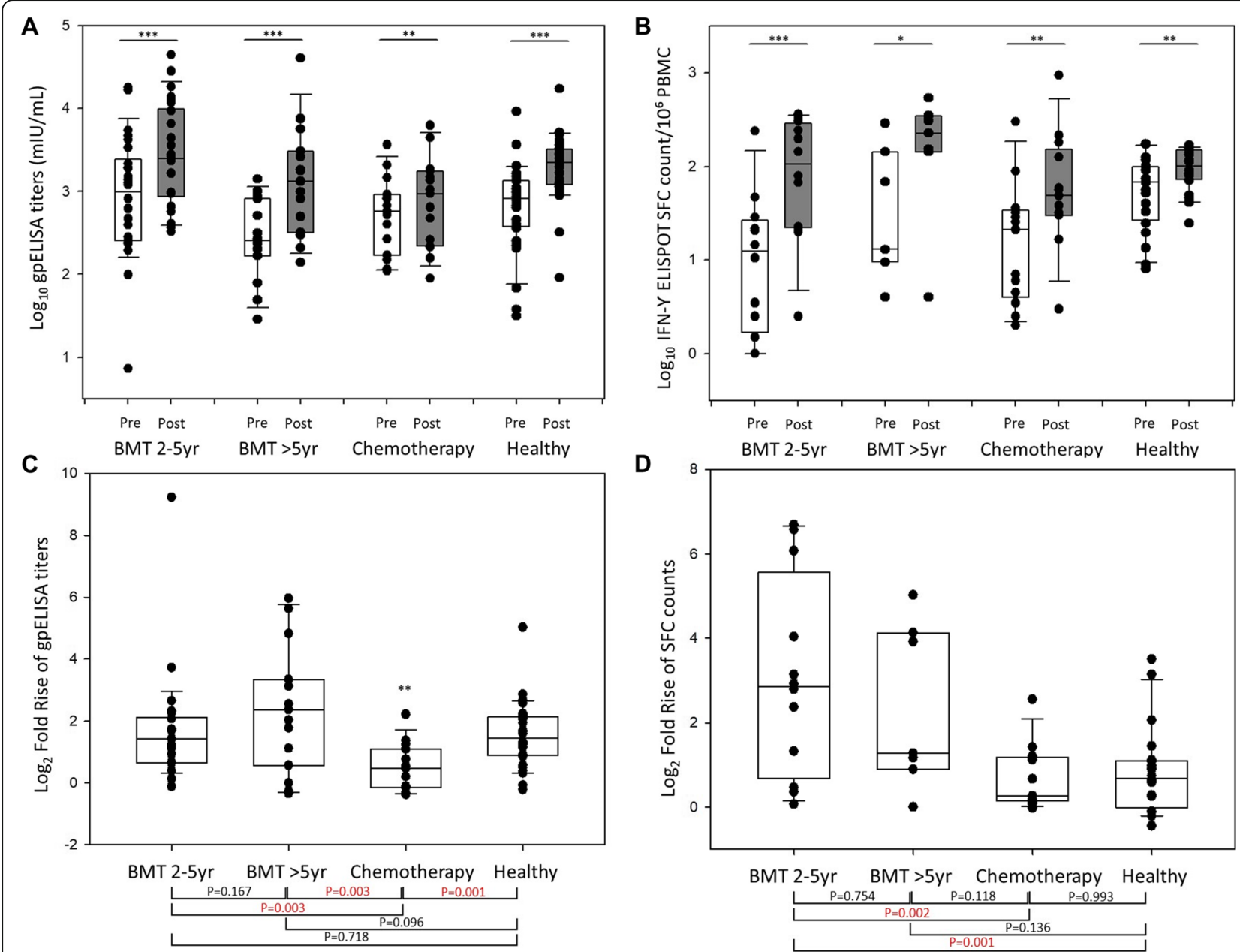

Fig. 2 Varicella-zoster virus-specific humoral immune responses measured in a glycoprotein ELISA (gpELISA) assay before and at 6 weeks postvaccination (a) and Log $_{2}$-fold increases in the gpELISA titers (c) for each study group. Varicella-zoster virus-specific cellular immune responses,

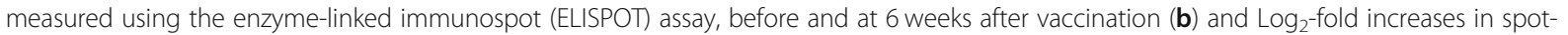
forming cell (sfc) counts (d) for each study group. The edges of the boxes represent the 25th and 75th percentiles, the horizontal lines inside the boxes are the median values, and the vertical whiskers represent the 2.5 th and 97.5 th percentiles $\left({ }^{*} p<0.05,{ }^{* *} p<0.01,{ }^{* * *} p<0.001\right)$

slowly reconstituted. Here, we demonstrated that a live $\mathrm{HZ}$ vaccine could be administered safely to hematologic malignancy patients, and that it induced humoral and cellular immune responses as strong as those in healthy individuals. A previous study examined HZ vaccineinduced immunity in the general population, and we found that immune responses in that study were similar to those of the healthy group in this study, which raises the reliability of our study [23].

Regarding the timing of vaccination, both HSCT 2-5 yr and HSCT $>5$ yr groups had comparable immune responses after $\mathrm{HZ}$ vaccination. Moreover, the baseline gpELISA-determined GMT was much lower in HSCT > $5 \mathrm{yr}$ than any other group, highlighting the need for prompt vaccination of HSCT recipients. Interestingly, vaccine-induced humoral and cellular GMFR responses in the HSCT groups were higher than in the chemotherapy group. We also have reported that influenza vaccination induced higher immune responses in cancer patients receiving immune checkpoint inhibitors than those receiving cytotoxic chemotherapy $[24,25]$.

We further explored the immune responses with respect to whether or not the participant had a history of shingles. Overall, the baseline gpELISA-determined GMTs and ELISPOT-determined GMCs were higher in patients with a history of shingles than those without. ELISPOT-determined GMCs did not exhibit consistent results, which could be attributed to the small number of samples. Nonetheless, GMFRs of humoral and cellular immune responses were uninfluenced by a history of shingles.

Two previous studies of $\mathrm{HZ}$ vaccines administered at a median 21-27 months post-transplantation reported that it was safe, and that it reduced the incidence of VZV 
Table 2 Adverse Events

\begin{tabular}{|c|c|c|c|c|}
\hline & $\begin{array}{l}\text { HSCT 2-5 yr } \\
(n=26)\end{array}$ & $\begin{array}{l}\text { HSCT > 5 yr } \\
(n=15)\end{array}$ & $\begin{array}{l}\text { Chemotherapy } \\
(n=15)\end{array}$ & $\begin{array}{l}\text { Healthy } \\
(n=30)\end{array}$ \\
\hline Any adverse events, n (\%) & $5(19.2)$ & $3(20.0)$ & $2(13.3)$ & $0(0)$ \\
\hline Local adverse events, n (\%) & $4(15.4)$ & $2(13.3)$ & $1(6.6)$ & $0(0)$ \\
\hline Pain or tenderness & $3(11.5)$ & $2(13.3)$ & $1(6.6)$ & $0(0)$ \\
\hline Redness & $3(11.5)$ & $2(13.3)$ & $1(6.6)$ & $0(0)$ \\
\hline Induration or edema & $1(3.8)$ & $2(13.3)$ & $1(6.6)$ & $0(0)$ \\
\hline Itching & $3(11.5)$ & $2(13.3)$ & $1(6.6)$ & $0(0)$ \\
\hline Systemic adverse events, n (\%) & $3(11.5)$ & $1(6.6)$ & $1(6.6)$ & $0(0)$ \\
\hline Headache & $1(3.8)$ & $1(6.6)$ & $0(0)$ & $0(0)$ \\
\hline Myalgia or arthralgia & $3(11.5)$ & $1(6.6)$ & $0(0)$ & $0(0)$ \\
\hline Fatigue & $3(11.5)$ & $0(0)$ & $1(6.6)$ & $0(0)$ \\
\hline Fever & $0(0)$ & $0(0)$ & $0(0)$ & $0(0)$ \\
\hline Systemic allergic reaction & $0(0)$ & $0(0)$ & $0(0)$ & $0(0)$ \\
\hline Nausea or vomiting & $0(0)$ & $0(0)$ & $0(0)$ & $0(0)$ \\
\hline Diarrhea & $0(0)$ & $0(0)$ & $0(0)$ & $0(0)$ \\
\hline
\end{tabular}

HSCT Hematopoietic stem cell transplantation, $N$ Number

infection $[4,26]$. Here, we found no VZV reactivation after administration of a live $\mathrm{HZ}$ vaccine to patients with a hematologic malignancy. Vaccine-related adverse reactions, both local and systemic, were more common in patients than in healthy volunteers; however, none were severe. Patients with hematologic malignancy might be more sensitive than healthy volunteers to adverse events and more prone to report mild symptoms. All patients recovered spontaneously within 1 week after immunization.

The study has some limitations. First, fewer patients qualified for the ELISPOT assay than for the gpELISA due to lack of sufficient blood. Inevitably, this led to less reliable results in terms of cellular immune responses. Second, patients in the HSCT $>5$ yr group had fewer members that the other groups; this was due to survival and recurrence rates, chronic GvHD, and other medical issues. Third, we monitored vaccine adverse events until 6 weeks post-vaccination; a longer follow-up time is considered better for monitoring the adverse events of live vaccines, although life-threatening allergic reactions tend to appear shortly after vaccination [27].

Two inactive $\mathrm{HZ}$ vaccines have been introduced recently, and the published safety and efficacy data seem very strong $[28,29]$. Unfortunately however, neither vaccine is available in most countries, including South Korea. Further studies of cost-effectiveness are needed. In practical terms, we expect to continue using live VZV vaccines (at least for a few years); therefore, the immunogenicity and safety data presented herein will be useful in clinical practice.

\section{Conclusions}

In summary, the $\mathrm{HZ}$ vaccine could induce both humoral and cellular immune responses in patients undergoing
HSCT, comparable to those in healthy volunteers. Hematologic malignancy patients who were undergoing cytotoxic chemotherapy showed weaker immune responses against the $\mathrm{HZ}$ vaccine. There was no reactivation of VZV during follow-up (up to 6 weeks postimmunization), and all reported adverse events were mild. These findings support current guidelines stating that live $\mathrm{HZ}$ vaccines may be administered to patients 2 years post-HSCT [9-11].

\section{Supplementary Information}

The online version contains supplementary material available at https://doi. org/10.1186/s12879-021-05806-4.

Additional file 1: Supplementary Table 1. Humoral and cellular responses after Herpes Zoster vaccination. Supplementary Table 2. Humoral responses after Herpes Zoster vaccination by shingles history. Supplementary Table 3. Cellular responses after Herpes Zoster vaccination by shingles history.

\section{Abbreviations}

HZ: Herpes zoster; HSCT: Hematopoietic stem cell transplant; VZV: Varicella zoster virus; GvHD: Graft-versus-host disease; gpELISA: Glycoprotein enzymelinked immunosorbent assay; ELISPOT: Interferon- $\gamma$ (IFN- $\gamma$ ) enzyme-linked immunospot; GMFR: Geometric mean fold rise; GMT: Geometric mean titers; GMC: Geometric mean concentrations; Cl: Confidence interval; CoP: Correlates of protection; IgGs: Immunoglobulin G antibodies; PBMCs: Peripheral blood mononuclear cells; SFC: Spot-forming cells; IQR: Interquartile range

\section{Acknowledgements}

We thank Su-Jin Choi (Laboratory of Infection \& Immunity, Seoul National University Hospital Biomedical Research Institute, Seoul, Korea) for technical assistance.

Authors' contributions

WBP and IHK designed the study protocol. JYC, KCK, MKL, CKK, YIK, DYS, JSH and SSY participated in the data collection and the interpretation of data. PGC, NJK and MDO advised on data analysis and interpretation. JYC and KCK 
drafted the manuscript. WBP revised the manuscript and finalized it. All authors read and approved the final manuscript.

\section{Funding}

This study was supported by the SNUH Research Fund [grant no 03-20170360]. The Funders had no role in study design and collection, analysis or interpretation of the data.

\section{Availability of data and materials}

All included data are available from the corresponding author upon request.

\section{Ethics approval and consent to participate}

The study was approved by the Institutional Ethics Review Board of Seoul National University Hospital (No. 1705-030-852). All subjects who participated in the study supplied informed written consent. The informed consent form and study-related samples were collected under the permission of above committee (Institutional Review Board, SNUH, Seoul, South Korea). Data used in this study was anonymized before its use.

\section{Consent for publication}

Not applicable.

\section{Competing interests}

The authors declare no competing interests.

\section{Author details}

${ }^{1}$ Department of Internal Medicine, Seoul National University College of Medicine, 101 Daehak-ro, Jongno-gu, Seoul 03080, Republic of Korea. ${ }^{2}$ Present affiliation: Department of Internal Medicine, National Cancer Center, Goyang, South Korea.

Received: 23 September 2020 Accepted: 15 January 2021

Published online: 26 January 2021

\section{References}

1. Levin MJ. 65 - zoster vaccines. In: Plotkin SA, Orenstein WA, Offit PA, Edwards KM, editors. Plotkin's vaccines (seventh edition): Elsevier; 2017. p. 1268-1281.e1266.

2. Kasper D, Fauci A, Hauser S, Longo D, Jameson J, Loscalzo J. Harrison's principles of internal medicine, 19e; 2015.

3. Bastidas A, de la Serna J, El Idrissi M, Oostvogels L, Quittet P, Lopez-Jimenez $J$, et al. Effect of recombinant zoster vaccine on incidence of herpes zoster after autologous stem cell transplantation: a randomized clinical trial. Jama. 2019:322(2):123-33.

4. Issa NC, Marty FM, Leblebjian H, Galar A, Shea MM, Antin JH, et al. Live attenuated varicella-zoster vaccine in hematopoietic stem cell transplantation recipients. Biol Blood Marrow Transpl. 2014;20(2):285-7.

5. Su SH, Martel-Laferriere V, Labbe AC, Snydman DR, Kent D, Laverdiere M, et al. High incidence of herpes zoster in nonmyeloablative hematopoietic stem cell transplantation. Biol Blood Marrow Transpl. 2011;17(7):1012-7.

6. Wang $L$, Verschuuren EAM, van Leer-Buter CC, Bakker SJL, de Joode AAE, Westra J, et al. Herpes zoster and immunogenicity and safety of zoster vaccines in transplant patients: a narrative review of the literature. Front Immunol. 2018:9:1632.

7. Costa E, Buxton J, Brown J, Templeton KE, Breuer J, Johannessen I. Fatal disseminated varicella zoster infection following zoster vaccination in an immunocompromised patient. BMJ Case Rep. 2016;2016:bcr2015212688. https://doi.org/10.1136/bcr-2015-212688.

8. Croce E, Hatz C, Jonker EF, Visser LG, Jaeger VK, Bühler S. Safety of live vaccinations on immunosuppressive therapy in patients with immunemediated inflammatory diseases, solid organ transplantation or after bonemarrow transplantation - a systematic review of randomized trials, observational studies and case reports. Vaccine. 2017:35(9):1216-26.

9. Harpaz R, Ortega-Sanchez IR, Seward JF. Prevention of herpes zoster: recommendations of the advisory committee on immunization practices (ACIP). MMWR Recomm Rep. 2008;57(Rr-5):1-30 quiz CE32-34.

10. Kroger AT, Atkinson WL, Marcuse EK, Pickering LK. General recommendations on immunization: recommendations of the advisory committee on immunization practices (ACIP). MMWR Recomm Rep. 2006; 55(Rr-15):1-48.
11. Guidelines for preventing opportunistic infections among hematopoietic stem cell transplant recipients. MMWR Recomm Rep. 2000;49(Rr-10):1-125 ce121-127.

12. Cordonnier C, Einarsdottir S, Cesaro S, Di Blasi R, Mikulska M, Rieger C, et al. Vaccination of haemopoietic stem cell transplant recipients: guidelines of the 2017 European conference on infections in Leukaemia (ECIL 7). Lancet Infect Dis. 2019;19(6):e200-12.

13. Thomson KJ, Hart DP, Banerjee L, Ward KN, Peggs KS, Mackinnon S. The effect of low-dose aciclovir on reactivation of varicella zoster virus after allogeneic haemopoietic stem cell transplantation. Bone Marrow Transplant. 2005;35(11):1065-9.

14. Ljungman $P$, Engelhard D, de la Camara R, Einsele H, Locasciulli A, Martino $\mathrm{R}$, et al. Vaccination of stem cell transplant recipients: recommendations of the infectious diseases working party of the EBMT. Bone Marrow Transplant. 2005;35(8):737-46.

15. Gilbert PB, Gabriel EE, Miao X, Li X, Su SC, Parrino J, et al. Fold rise in antibody titers by measured by glycoprotein-based enzyme-linked immunosorbent assay is an excellent correlate of protection for a herpes zoster vaccine, demonstrated via the vaccine efficacy curve. J Infect Dis. 2014;210(10):1573-81.

16. Krah DL, Cho I, Schofield T, Ellis RW. Comparison of gpELISA and neutralizing antibody responses to Oka/Merck live varicella vaccine (Varivax) in children and adults. Vaccine. 1997;15(1):61-4.

17. Wasmuth EH, Miller WJ. Sensitive enzyme-linked immunosorbent assay for antibody to varicella-zoster virus using purified VZV glycoprotein antigen. J Med Virol. 1990;32(3):189-93.

18. Smith JG, Liu X, Kaufhold RM, Clair J, Caulfield MJ. Development and validation of a gamma interferon ELISPOT assay for quantitation of cellular immune responses to varicella-zoster. Virus. 2001;8(5):871-9.

19. Smith JG, Liu X, Kaufhold RM, Clair J, Caulfield MJ. Development and validation of a gamma interferon ELISPOT assay for quantitation of cellular immune responses to varicella-zoster virus. Clin Diagn Lab Immunol. 2001;8(5):871-9.

20. Kim JW, Min CK, Mun YC, Park Y, Kim BS, Nam SH, et al. Varicella-zoster virus-specific cell-mediated immunity and herpes zoster development in multiple myeloma patients receiving bortezomib- or thalidomide-based chemotherapy. J Clin Virol. 2015;73:64-9.

21. Food US, Administration D. Guidance for industry: toxicity grading scale for healthy adult and adolescent volunteers enrolled in preventive vaccine clinical trialsIn. Edited by US Food and Drug Administration; 2007.

22. Tozzi AE, Asturias EJ, Balakrishnan MR, Halsey NA, Law B, Zuber PL. Assessment of causality of individual adverse events following immunization (AEFI): a WHO tool for global use. Vaccine. 2013;31(44):5041-6.

23. Levin MJ, Oxman MN, Zhang JH, Johnson GR, Stanley H, Hayward AR, et al. Varicella-zoster virus-specific immune responses in elderly recipients of a herpes zoster vaccine. J Infect Dis. 2008;197(6):825-35.

24. Keam B, Kang CK, Jun Kl, Moon SM, Suh KJ, Lee DW, et al. Immunogenicity of influenza vaccination in patients with cancer receiving immune checkpoint inhibitors. Clin Infect Dis. 2020;71(2):422-5.

25. Kang CK, Kim HR, Song KH, Keam B, Choi SJ, Choe PG, et al. Cell-mediated immunogenicity of influenza vaccination in patients with cancer receiving immune checkpoint inhibitors. J Infect Dis. 2020;222(11):1902-9.

26. Aoki T, Koh K, Kawano Y, Mori M, Arakawa Y, Kato M, et al. Safety of live attenuated high-titer varicella-zoster virus vaccine in pediatric allogeneic hematopoietic stem cell transplantation recipients. Biol Blood Marrow Transpl. 2016:22(4):771-5.

27. General Best Practice Guidelines for Immunization: Best Practices Guidance of the Advisory Committee on Immunization Practices (ACIP) [https://www. cdc.gov/vaccines/hcp/acip-recs/general-recs/adverse-reactions.html]. Accessed 17 Dec 2020

28. Winston DJ, Mullane KM, Cornely OA, Boeckh MJ, Brown JW, Pergam SA, et al. Inactivated varicella zoster vaccine in autologous haemopoietic stemcell transplant recipients: an international, multicentre, randomised, doubleblind, placebo-controlled trial. Lancet (London, England). 2018;391(10135): 2116-27.

29. Dagnew AF, Ilhan O, Lee WS, Woszczyk D, Kwak JY, Bowcock S, et al. Immunogenicity and safety of the adjuvanted recombinant zoster vaccine in adults with haematological malignancies: a phase 3, randomised, clinical trial and post-hoc efficacy analysis. Lancet Infect Dis. 2019;19(9):988-1000.

\section{Publisher's Note}

Springer Nature remains neutral with regard to jurisdictional claims in published maps and institutional affiliations. 